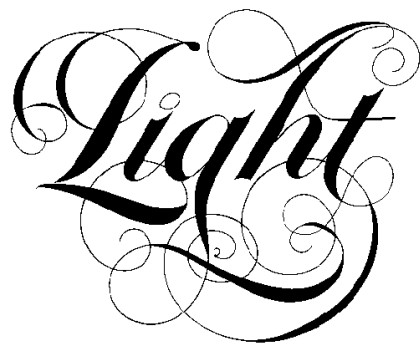

\title{
Efficient Single-frequency Fibre Lasers with Novel Photosensitive Er/Yb Optical Fibers
}

\author{
L.Dong, W.H.Loh, J.E.Caplen and J.D.Minelly, *K.Hsu and L.Reekie
}

Micron Optics, Inc., Suite 140, 2801 Buford Highway, Atlanta, Georgia 30329

\begin{abstract}
Boron- and germanium-doped highly photosensitive cladding is used in a novel design to achieve photosensitive Er/Yb-doped fibers, permitting short, strong gratings (length $\sim 1 \mathrm{~cm}$, reflectivity $>99 \%$ ) to be written without hydrogenation. The high absorption at $980 \mathrm{~nm}$ in $\mathrm{Er} / \mathrm{Yb}$ fibers permits efficient pump absorption over a short device length, which is ideal for achieving highly efficient single-frequency fiber lasers. Both single-frequency Bragg-grating reflector and distributed-feedback lasers with slope efficiencies of $25 \%$ with respect to launched pump power have been realized in such fibers.
\end{abstract}

Single-frequency fiber lasers using UV-written fiber Bragg gratings are a promising competing technology for semiconductor distributed-feedback (DFB) lasers for use in optical fiber telecommunications as well as sensor systems. Single-frequency fiber lasers are potentially inexpensive, yield high performance, and are tunable over the whole Er band, as has been demonstrated.[1-3] Two types of fiber have been used to construct such lasers: Er-doped germanosilicate fibers and $\mathrm{Er} / \mathrm{Yb}$-doped phosphosilicate fibers. In an Er/Yb-doped fiber, the 980nm pump energy is efficiently absorbed by the strong $\mathrm{Yb}$ absorption over a length of just a few centimeters and subsequently transferred to the Er. The 2-orders-of-magnitude increase in the pump absorption, which is much greater than that of Er-doped fibers, allows the realization of highly efficient short-cavity lasers. With Er-doped fibers, however, large amounts of germanium (>20mol.\%) can be incorporated into the core, rendering it photosensitive enough for easy realization of strong Bragg gratings. This is unfortunately not possible with Er/Yb-doped fibers, as a phosphosilicate glass host needs to be used to ensure efficient energy transfer between the $\mathrm{Er}$ and $\mathrm{Yb}$ ions. Phosphorous doping is known to reduce fiber photosensitivity even when some germanium can be incorporated. One can make Er/Yb distributed Bragg reflector (DBR) singlefrequency lasers by splicing the doped fiber to fiber Bragg gratings [2]; however, intracavity splice losses are an obvious concern. $\mathrm{Er} / \mathrm{Yb}$ DFB lasers have also been made by hydrogenation [4] and recently with Sn codoping [5]. As we have observed [5], significant loss at the pump wavelength arising from a broad UV absorption band can be induced when hydrogenation is used, lowering the laser efficiency (Fig. 1 shows details of induced loss observed in various fiber hosts). In particular, UV exposure of hydrogenated fibers results in substantial $\mathrm{OH}$ formation, with associated losses near $0.95 \mu \mathrm{m}$ [6], that is resistant to annealing. On the other hand, Sn codoping has permitted the implementation of a $10 \mathrm{~cm}$-long DFB laser with $11 \%$ slope efficiency, without the need to resort to hydrogenation. Shorter devices, which are

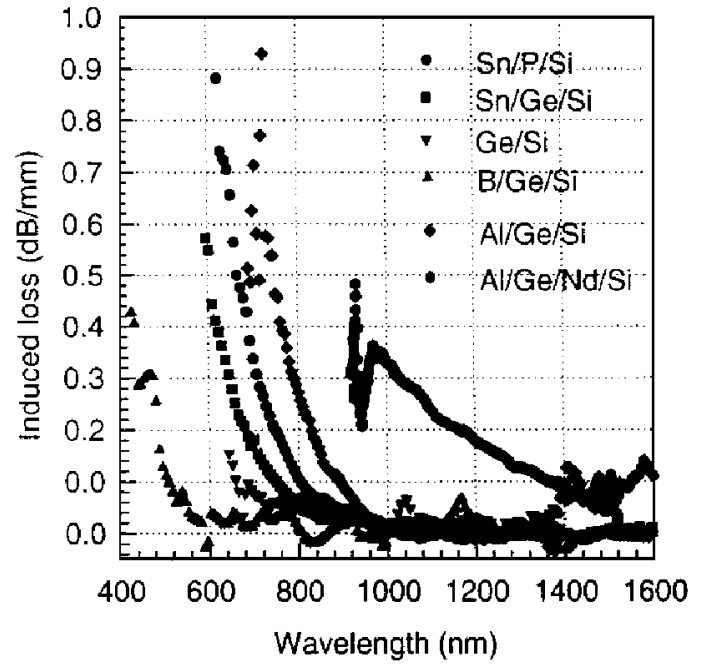

Fig. 1. UV-induced absorption in various types of fiber. A $248 \mathrm{~nm}$ excimer laser was used as the UV source, operating at $0.3 \mathrm{~J} / \mathrm{cm}^{2}$ and $20 \mathrm{~Hz}$. The exposure time is $\mathbf{5}$ min. 
desirable from a practical point of view, are, however, still difficult to realize without further improvement in the photosensitivity of such fibers. A highly photo-sensitive $\mathrm{Er} / \mathrm{Yb}$ fiber is therefore much needed, as its absence has hampered the progress of a variety of devices that could be based on such a fiber.

In this Letter we demonstrate a simple and efficient method to achieve highly photosensitive $\mathrm{Er} / \mathrm{Yb}$ fibers that requires no modification of the host glass for $\mathrm{Er}$ and $\mathrm{Yb}$ ions or compromise on the overlap of the rare-earth dopants with the guided optical field. In this approach a highly photosensitive $\mathrm{B} / \mathrm{Ge}$-doped silica cladding is used to surround a standard $\mathrm{Er} / \mathrm{Yb}$ doped phosphosilicate core (Fig. 2). The germanium doping increases the refractive index of silica, even though the boron doping lowers it, so the same refractive index as that of pure silica can be achieved for the cladding. The $\mathrm{Er} / \mathrm{Yb}$-doped core is not affected by the $\mathrm{B} / \mathrm{Ge}$-doped silica cladding. The highly photosensitive cladding allows strong gratings (>99\%) $1 \mathrm{~cm}$-long to be easily achieved in these

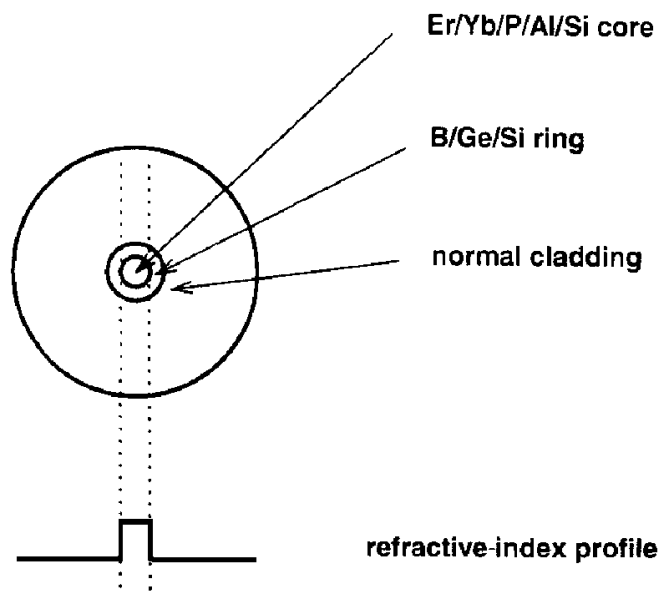

Fig. 2. Structure of the photosensitive Er/Yb fiber with an index-matched $\mathrm{B} / \mathrm{Ge} / \mathrm{Si}$ cladding.

fibers, despite the reduced overlap between the grating and the guided optical field. We have constructed single-frequency fiber lasers of both DBR and DFB designs with better than $20 \%$ slope efficiency in these fibers. We have also achieved submilliwatt thresholds, demonstrating the low cavity loss achievable with this type of fiber.

A number of fibers were fabricated for this study by a modified chemical-vapordeposition setup in combination with the solution-doping technique. Two layers of B/Ge-doped silica with a refractive index matched to that of the silica substrate were deposited before the phosphosilicate core was deposited at $1100^{\circ} \mathrm{C}$ with the burner traversing upstream from the precursor vapor flow to ensure that the core was not sintered. A methanol solution of $\mathrm{ErCl}_{3}, \mathrm{YbCl}_{3}$, and $\mathrm{AlCl}_{3}$ was used for the solution doping. A 30 minute drying procedure was then performed before the core was sintered. By separating the photosensitive region from the $\mathrm{Er} / \mathrm{Yb}$ core, we avoid perturbing the composition for efficient energy transfer between $\mathrm{Er}$ and $\mathrm{Yb}$ ions. The overlap

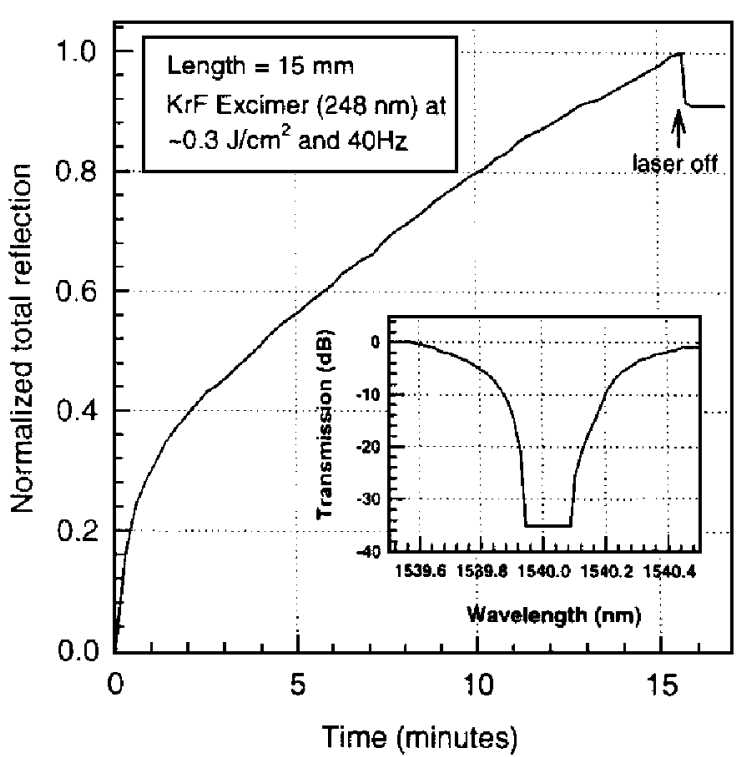

Fig. 3. Grating formation in the photosensitive $\mathrm{Er} / \mathrm{Yb}$ fiber. 
between the guided optical field and the rare-earth dopants is not affected either, ensuring maximum pump absorption and gain per unit of fiber length. B/Ge-doped silica has demonstrated photoinduced index changes as great as $2 \times 10^{-3}$ [7], which, accounting for the reduced overlap between the guided optical field and the photosensitive region,

should give effective index changes of $>10^{-4}$ for fibers with $\mathrm{V}$ values between 1.5 and 2.4, enough for short, strong Bragg gratings. Figure 3 shows the growth of the total reflection and final transmission spectrum of a $1.5 \mathrm{~cm}$-long grating written in such a fiber. The grating was written with an interferometer by a linenarrowed $\mathrm{KrF}$ excimer laser. The FWHM bandwidth of the grating is $0.58 \mathrm{~nm}$, giving an effective index modulation of $5 \times 10^{-4}$. A typical $\mathrm{Er} / \mathrm{Yb}$-doped phosphosilicate core has a small signal absorption of $230 \mathrm{~dB} / \mathrm{m}$ at $980 \mathrm{~nm}$, an $\mathrm{Er}^{3+}$ absorption of $25 \mathrm{~dB} / \mathrm{m}$ at $1535 \mathrm{~nm}$, a cutoff wavelength of $1470 \mathrm{~nm}$, and a numerical aperture (NA) of 0.18 .

A DBR laser with the configuration shown in Fig. 4 was constructed. A standard telecommunications fiber (0.12 NA, $1250 \mathrm{~nm}$ cutoff wavelength) was spliced to the $\mathrm{Er} / \mathrm{Yb}$ fiber. A $5 \mathrm{~mm}$-long grating output coupler ( $90 \%)$ was written next to the splice, and then a $1 \mathrm{~cm}$-long high reflector $(\sim$ $99 \%$ ) was written $2 \mathrm{~cm}$ away from the output coupler. We monitored the transmission through the fiber during the grating writing to determine the reflectivity of the gratings. The $980 \mathrm{~nm}$ pump was coupled in through a wavelength-division-multiplexing coupler, and the laser output at $1535 \mathrm{~nm}$ was coupled out through the standard telecommunications fiber, which was in turn spliced to a pigtailed $1550 \mathrm{~nm}$ optical isolator to prevent feedback. The lasing characteristics of the DBR are shown in Fig. 4. The threshold is $4 \mathrm{~mW}$, and the slope efficiency is $\sim 25 \%$, after we take into consideration the $1 \mathrm{~dB}$ loss of the isolator and the $\sim 0.5 \mathrm{~dB}$ splice loss between the standard telecommunications fiber and the $\mathrm{Er} / \mathrm{Yb}$ fiber. The laser operates at a single frequency and a single

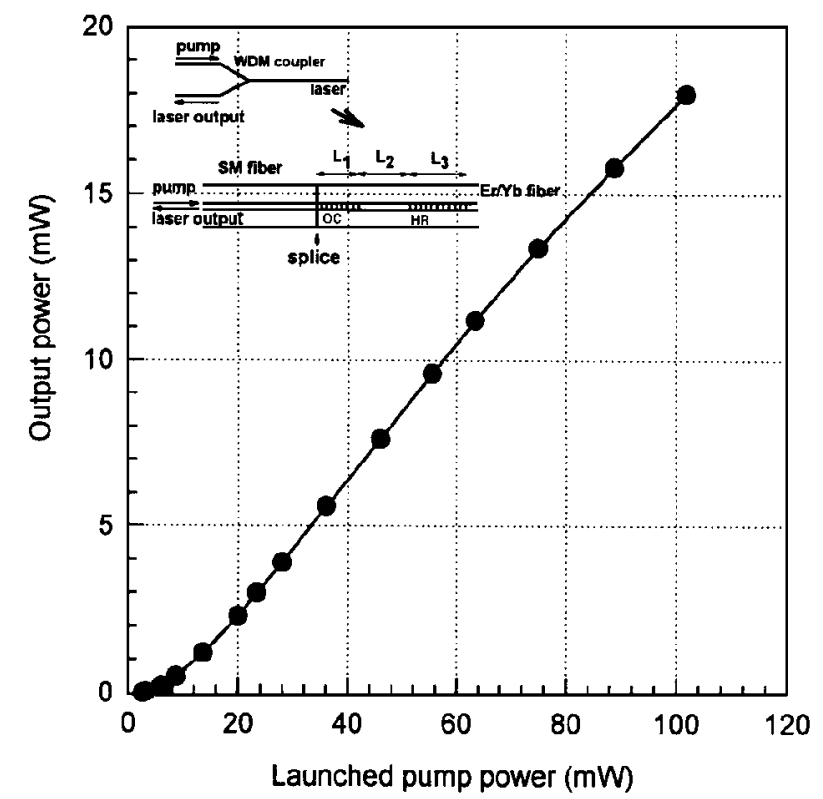

Fig. 4. DBR laser constructed with the photosensitive

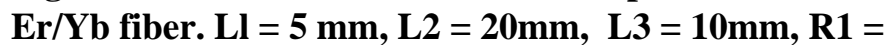
90\%, $\mathrm{R2}>99 \%$. WDM, wavelength division multiplexer; OC, output coupler; HR, high reflector.

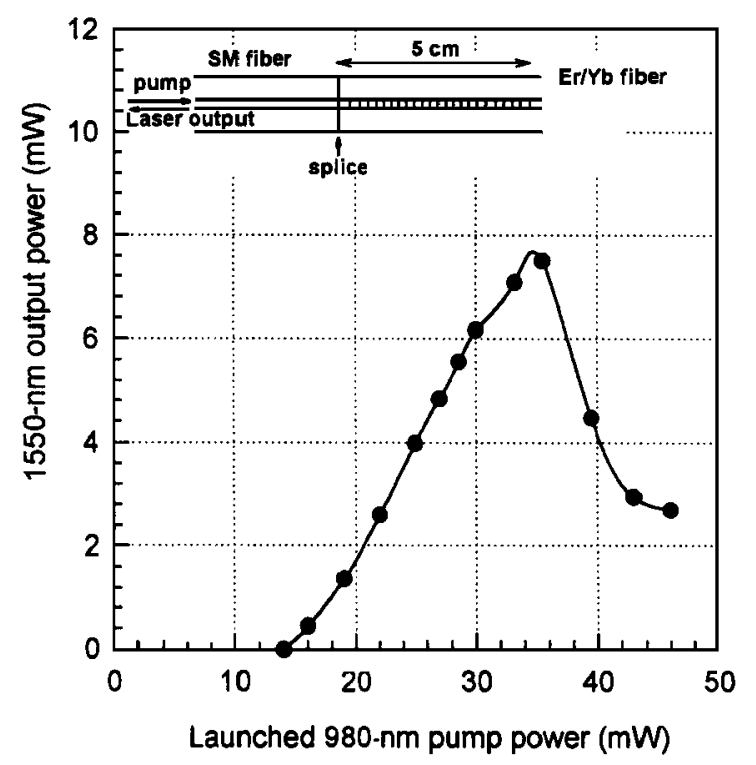

Fig. 5. DFB laser constructed with the photosensitive Er/Yb fiber. OC, output coupler; HR, high reflector; SM single mode. 
polarization for output power of as much as $8 \mathrm{~mW}$, beyond which the second polarization mode starts lasing as well. In another DBR laser with lower output coupling (higher reflectivity grating), the threshold was found to be submilliwatt, attesting to the low intrinsic losses in these laser cavities.

We also realized a DFB laser by writing a single $5 \mathrm{~cm}$-long grating in this fiber. We wrote the grating, centered at $1550.2 \mathrm{~nm}$, by scanning the UV beam from a frequency-doubled argon-ion laser across a phase mask. The laser performance is shown in Fig. 5. Despite the higher threshold $(14 \mathrm{~mW})$, the efficiency with respect to launched pump power is also $\sim 25 \%$, after we again take into consideration the $1 \mathrm{~dB}$ insertion loss of the isolator and the $0.5 \mathrm{~dB}$ splice loss between the standard telecommunication fiber and the $\mathrm{Er} / \mathrm{Yb}$ fiber. It is interesting that, as in the above DBR laser, single frequency and single-polarization operation were observed, with as much as $6 \mathrm{~mW}$ of output power. This is in contrast to the dual-polarization mode operation observed in previous lasers fabricated with the gratings in the core [4,5]. As singly polarized lasers are desirable for many applications, this photosensitive cladding design thus seems additionally attractive. The laser output was stable, with no spiking behaviour, and its suitability as a telecommunications source was verified in a $10 \mathrm{Gbit} / \mathrm{s}$ nonreturn to zero transmission system over $50 \mathrm{~km}$ of standard singlemode fiber. Comparison tests with a DFB semiconductor laser showed that the fiber laser transmitter performed equally well, with no penalties. For pump powers of $>30 \mathrm{~mW}$, however, the laser output was observed to decrease. Although the reason for this anomalous behaviour is not entirely clear, this problem is believed to be thermal, as the fall in output power occurs over a time scale of seconds, even though the pump power could be changed very quickly.

Finally, it is worth mentioning that the efficiencies of the DBR and DFB lasers constructed here are limited primarily by the efficiency of the fiber itself, which was determined to be $25 \%$ in a simple linear-cavity configuration, where the laser was constructed with a cleaved fiber and end face as one reflector, and the other fiber end butted against a high-reflectivity mirror. However, as slope efficiencies of as much as $40 \%$ were observed in previous (nonphotosensitive) $\mathrm{Er} / \mathrm{Yb}$ fibers, further improvements in the laser performance are expected by optimization of the $\mathrm{Er} / \mathrm{Yb}$ doped fiber core.

We have demonstrated efficient short-cavity DBR and DFB fiber lasers, using newly developed photosensitive-cladding Er/Yb fibers. Strong, short reflectors can be easily written in these fibers without resorting to hydrogenation, thus offering a potentially important technology for realizing efficient single-frequency fiber lasers. This technology can also be used to make other photosensitive fibers in which core materials cannot be made photosensitive.

\section{Acknowledgements}

This study was partially supported by the UK Department of Trade and Industry/Link Programme High Radiance Fibre Source and the European Community Advanced Communications Technologies and Services project Photosensitive Technology for Optical Systems. The Optoelectronics Research Centre is an interdisciplinary research center funded by the UK Engineering and Physical Sciences Research Council. 


\section{References}

1. G.Ball and W.W.Morey, Opt. Lett. 19, 1979 (1994).

2. J.T.Kringlebotn, J.L.Archambault, L.Reekie, J.E.Townsend, G.G.Vienne and D.N.Payne, Electron. Lett. 30, 972 (1994).

3. V.Mizrahi, D.J.DiGiovanni, R.M.Atkins, S.G.Grubb, Y.K.Park and J-M.P.Delavaux, J. Lightwave Technol. 11,2021 (1993).

4. J.T.Kringlebotn, J.L.Archambault, L.Reekie and D.N.Payne, Opt. Lett. 19, 2101 (1994).

5. $\quad$ W.H.Loh, L.Dong and J.E.Caplen, Appl. Phys. Lett. 69, 2151 (1996).

6. P.J.Lemaire, R.M.Atkins, V.Mizrahi and W.A.Reed, Electron. Lett. 29, 1191 (1993).

7. L.Dong, J.L.Cruz, L.Reekie, M.G.Xu and D.N.Payne, IEEE Photon. Technol. Lett. 7, 1048 (1995). 